\title{
Automatic Contours Detection in Myocardial GSPECT
}

\author{
L Comas, P Berthout, R Sabbah, O Blagosklonov, J Verdenet, M Baud, JC Cardot \\ Department of Nuclear Cardiology, Jean Minjoz Hospital, Besançon, France
}

\begin{abstract}
Myocardial gated SPECT is widely used to provide three-dimensional information on regional perfusion and function as well as left ventricular ejection fraction. In this study, we proposed a generic methodology using an edge detection technique based on the position and the shape of the myocardial wall on the scintigraphic images with no knowledge of counts statistics, axis, tracer distribution or artefacts. After thresholding, we obtained binary images. We applied morphological operations on these images to eliminate artefacts. These artefacts were due to abnormal tracer fixation. This procedure was performed on 110 explorations ( 8 time bins) and concerned the central scans of the 3 heart axes and the summed image.
\end{abstract}

\section{Introduction}

Acquisition of gated single-photon emission tomography of the myocardium (gSPECT) is routinely used in clinical practice to provide information on myocardial perfusion and contractile function [1]. After reconstruction, a rigorous left ventricular contouring on each cycle image and axis of the heart is required. We developed a program for automatic contour detection; it was applied without knowledge on counts statistics, chosen axis, tracer distribution or artefacts.

\section{Material and methods}

\subsection{Patients and image acquisition}

This study was performed on 110 explorations at stress ( 8 images per cardiac cycle) and concerned the central scans of the 3 heart axes and the summed image. $99 \mathrm{mTc}-$ gSPECT studies were performed in supine patients. The exercise test consisted of physical exercise combined with pharmacological vasodilatation. After exercise stress test, a dose of $15 \mathrm{MBq} / \mathrm{kg}$ of $99 \mathrm{mTc}$ was administered intravenously. A rotating dual-head gamma camera was used, equipped with low-energy high-resolution collimators; the energy window was centered on $140 \mathrm{keV}$ $\pm 15 \%$. The system detectors were at $90^{\circ}$. The gamma camera was rotated through a $180^{\circ}$ arc in a contour orbit around the patient's chest from $45^{\circ} \mathrm{RAO}$ at $6^{\circ}$ increments for $40-60 \mathrm{~s}$ each (32 views), 8 time bins [2]. 1.33 zoom was applied. Data were stored in a 64x64 matrix; the raw scintigraphic data were preprocessed with a backprojection algorithm and a Butterworth filter (frequency 0.25 , range 5) without attenuation correction was applied. Then, the series was smoothed and denoised using Karhunen-Loeve transform; we used the 3 first KLT images for reconstruction [3].

The processing was done using MATLAB version 5.3 (The MathWorks, inc.) on standard PC (PIII-733, 256Mb RAM)

\subsection{Properties}

The following topographical properties of the myocardial reconstructed images are involved:

- The heart takes more than a half of the image surface; with many pixels, a histogram analysis can be used.

- The resulting pictures are denoised, and the "useful" intensities are 5 to 10 times higher than the noise, both in rest and stress studies. This assumption justifies the thresholding.

- Polar representation of the contours is applicable because of the elliptical form of the left ventricle and its central position on the images.

- The artefacts correspond to some tissues with abnormal fixation of the pharmaceutical tracer, and they are outside of the cardiac zone. Morphological operations (erosion, dilation, skeleton) are therefore possible.

Skeletonization is a process for reducing foreground regions in a binary image to a skeletal remnant that largely preserves the extent and connectivity of the original region while eliminating most of the original foreground pixels.

The skeleton can be produced in two ways. The first is to use some kind of morphological thinning that sequentially erodes pixels from the boundary (while preserving the end points of line segments) until no more thinning is possible; at this point what is left approximates the skeleton. The alternative method is to first calculate the distance transform of the image. The distance transform is an operator whose resulting grey level image resembles the input image except where the grey level intensity of points inside foreground regions are changed, showing the distance to the closest boundary from each point. The skeleton then takes the shape of the singularities (i.e. creases or curvature discontinuities) in the distance transform [4-5]. 


\subsection{Image processing}

The contours procedure, we have developed, is a 4 steps technique :

Threshold : The heart contours are at one intensity which was between the median of the cumulated histogram (Figure 1) and 1/3 of this value (Figure 2).

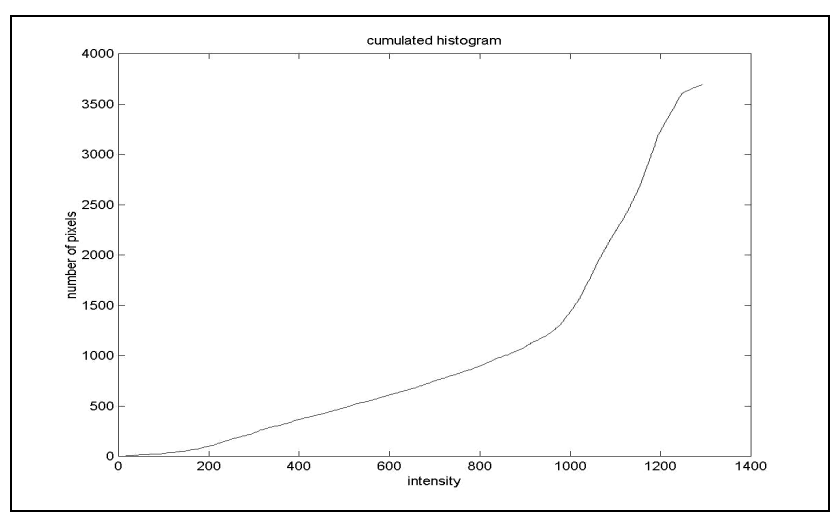

Figure 1. Cumulated histogram.

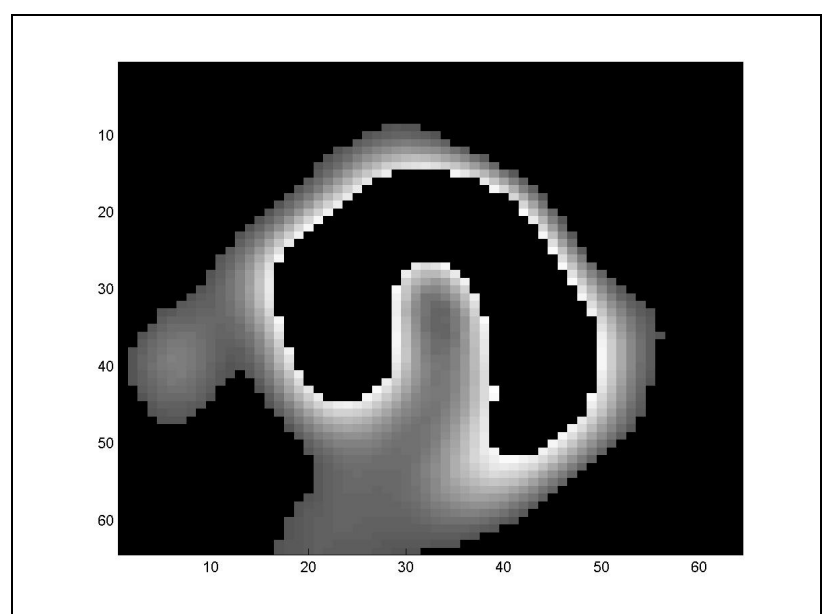

Figure 2. Threshold image : intensity between the median of the cumulated histogram and $1 / 3$ of this value was kept.

Then the profiles of intensity of 4 axes passing through the gravity center (horizontal, vertical and two diagonal) were calculated for this masked image (Figure 3 ); for each axis, we kept the intensity value just below the maximun when the peak (between 2 zeros) was larger than 2 pixels. Mean of these intensities was the best threshold for defining a image binary.

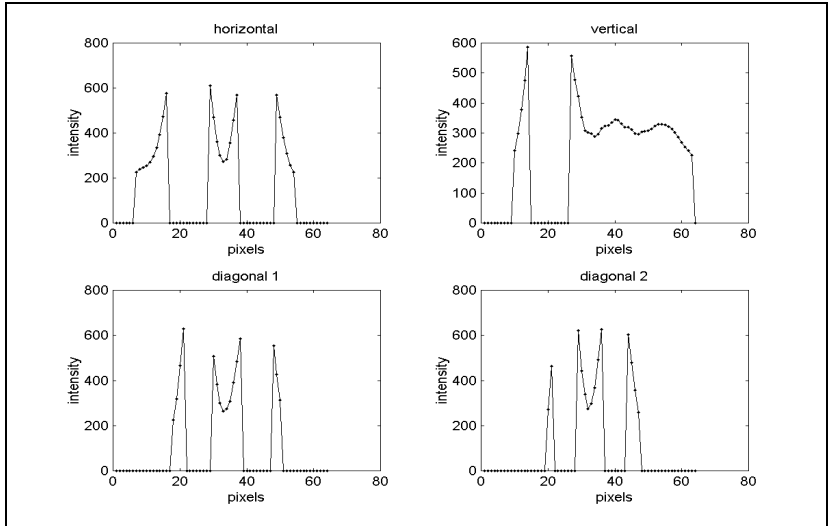

Figure 3. Profiles of intensity of 4 axes passing through the center of gravity

Detection of an isolated artefact, distant from heart areas (Figure 4): The largest zone is the heart. The whole heart is situated inside a circle whose center is the center of this zone and whose radius is the greatest distance of intersection between this zone and six specified axes (Figure 5). Areas outside of this circle were eliminated.

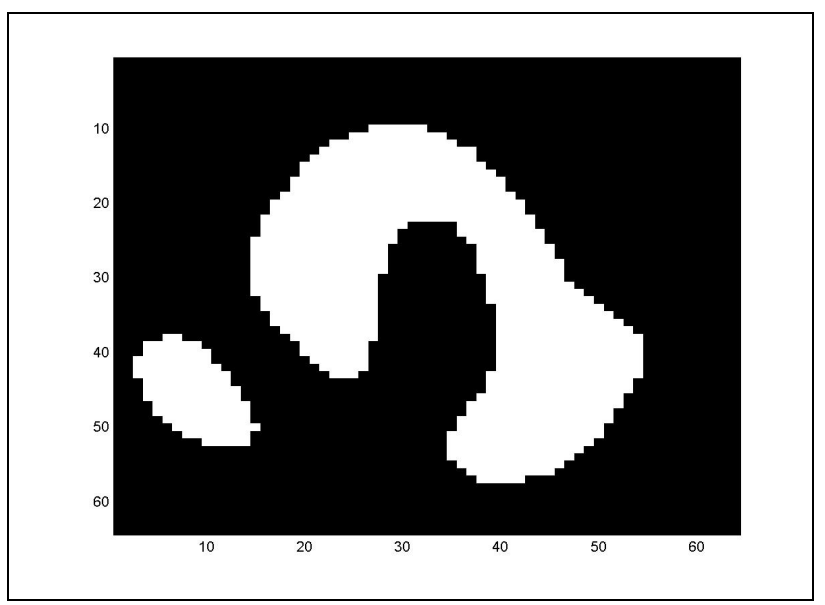

Figure 4. Isolated artefact far from heart areas

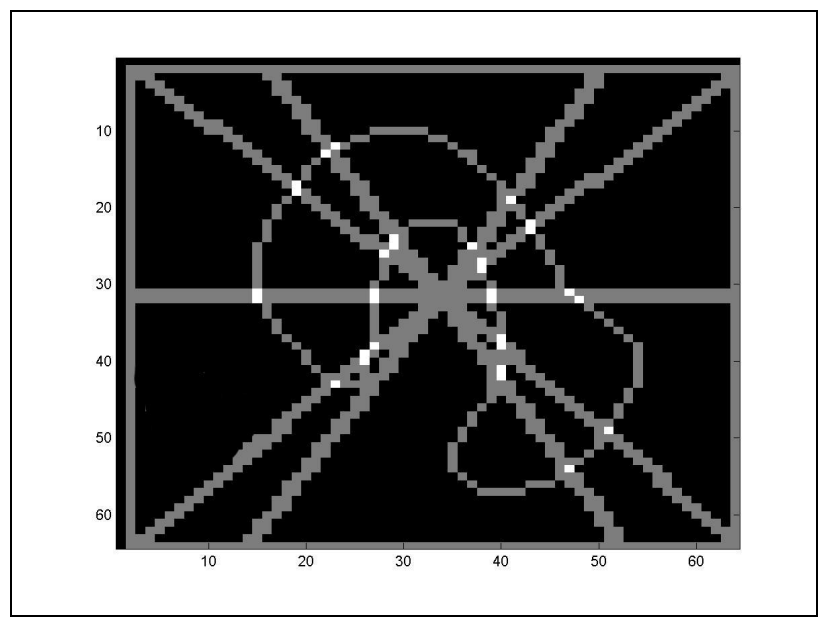

Figure 5. Zone with 6 specified axes 
Detection and elimination of background noise connected to the heart by a few pixels: These artefacts (Figure 6) were isolated after one or two erosions. They were eliminated using the method described in the above section.

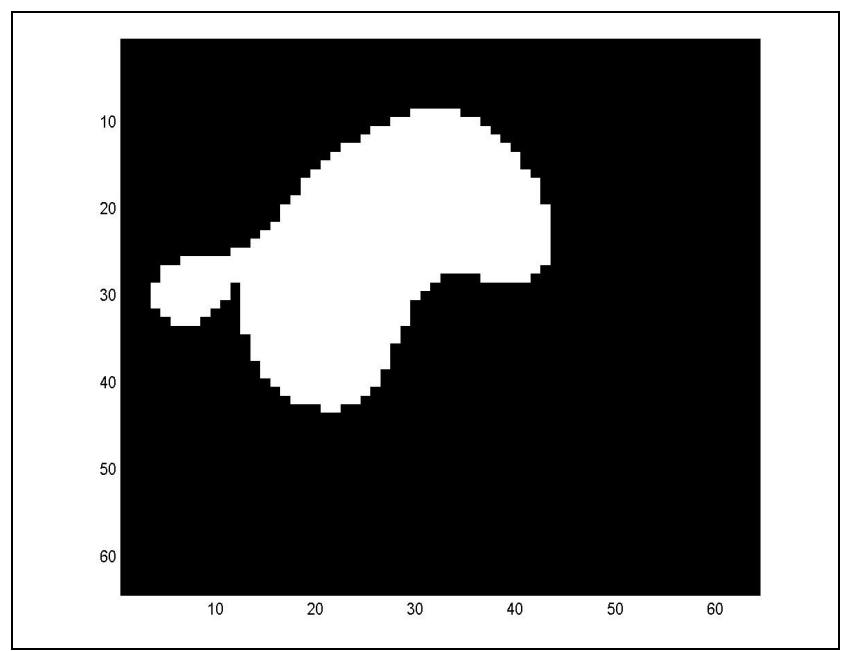

Figure 6. Background noise connected to the heart by a few pixels

Detection and elimination of background noise connected to the heart by many pixels: The skeleton of the heart (because of its form) looks like a ellipsis which can be open or closed. In the case of completely closed cavity, the skeleton is defined by half-maximum radii. Giving the imperfect elliptical form of the heart, some small branches on the skeleton can be observed (Figure 7). A noise region which is closely connected to the heart also induces a skeleton branch. The challenge was to identify these branches and eliminate the artifical ones.

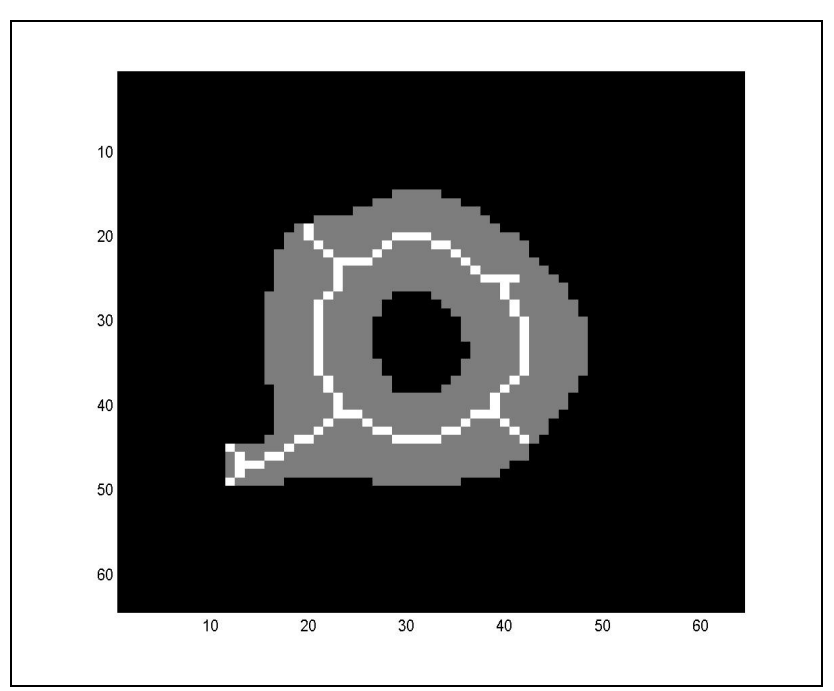

Figure 7. Region with some small branches on the skeleton
In polar coordinates (radius vs angle), the skeleton looks like a sinusoïdal curve. The additionnal branches generate picks (Figure 8a).

After a 3-points smoothing of the curve, we analysed the curve to detect radii greater than the mean radius. If the distance between two of these radii was greater than 1.44 pixels (squared cell), we eliminated the 2 nd radius and all the following points until the radius had the same intensity as the mean radius (Figure $8 \mathrm{~b}$ ).

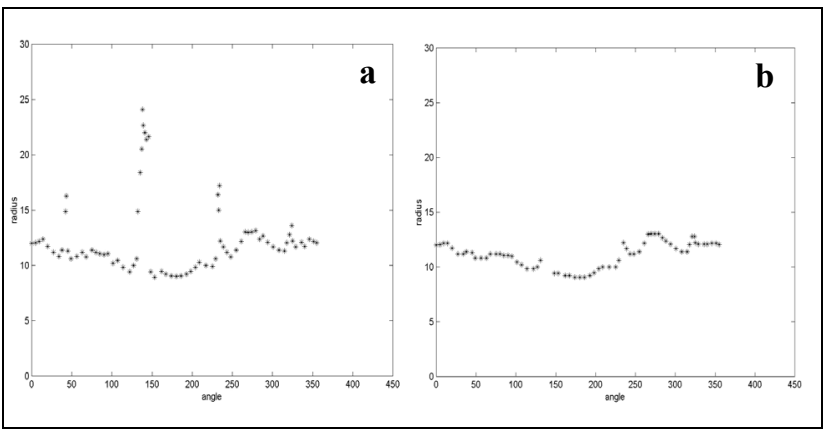

Figure 8. The skeleton in polar coordinates (a) before and (b) after processing

In Cartesian coordinates, we obtained the ebarbuled skeleton. The skeleton underwent $\mathrm{n}$ dilations (Figure 9). After each dilation, the correlation coefficient between this zone and the initial zone was calculated; the maximum correlation coefficient (Figure 10) corresponded to the number of dilations to be done. Those not appearing in the dilated skeleton but present in initial region zones were analyzed; for each one, we observed a zone of 3 pixels around the farest from the center point. If this zone reached the dilated skeleton, it was kept in the final zone (Figure 11).

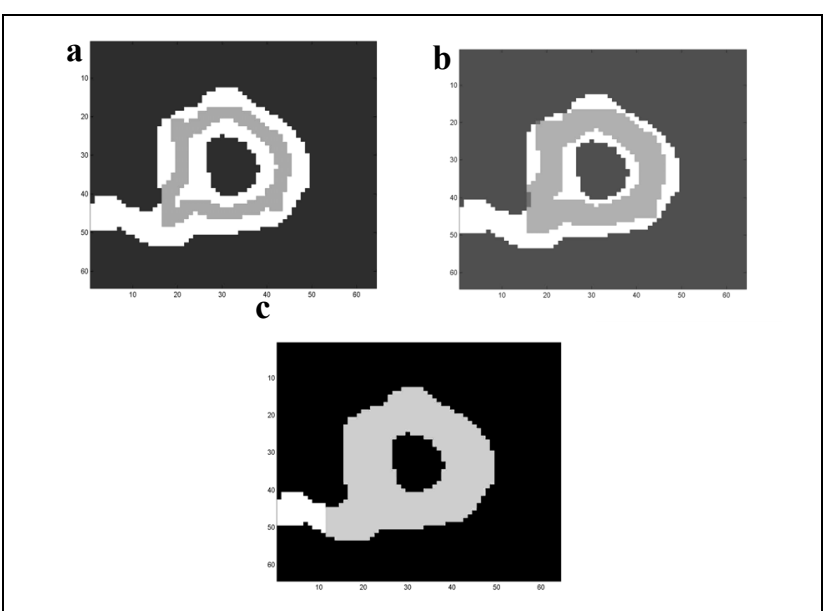

Figure 9. Skeleton after 1 dilation (a), 2 dilations (b) and 6 dilations (c) (white: initial ROI, gray:dilated skeleton, black:background) 


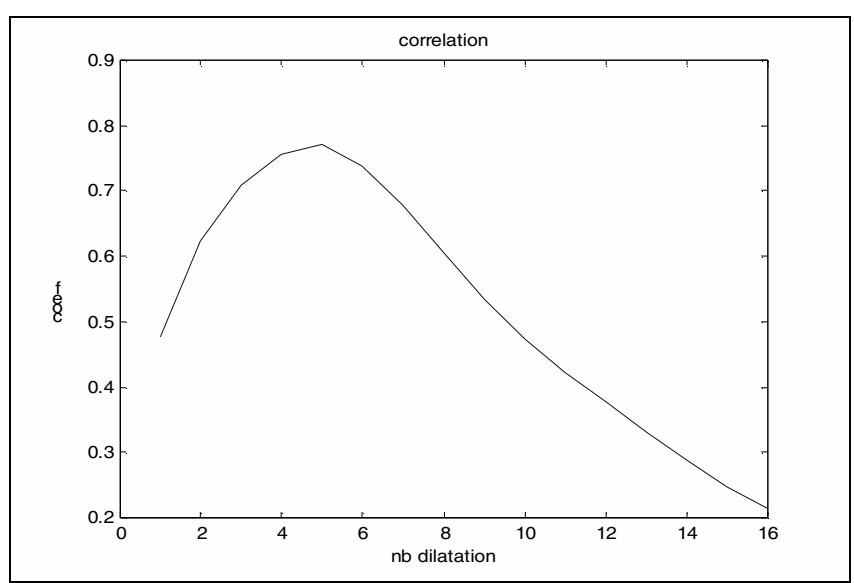

Figure 10. Correlation coefficient at each dilatation

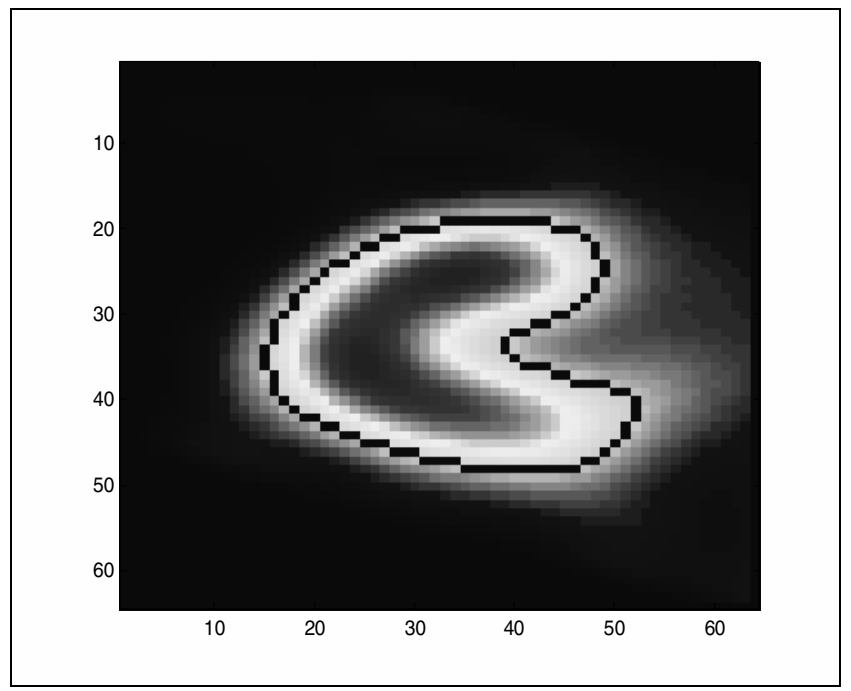

Figure 11. Resulting contour

\section{Results and discussion}

The method was tested on 2970 contours obtained from 110 explorations ( 3 axes*8 time bins+ mean image) acquired and reconstructed in two different hospital departments using different cameras (ADAC-GE) and processing software (SEGAMI-GE). Two different observers visually analysed the images with the contours. Analysis showed 92\% good results, 3\% acceptable results with small background zone less than 10 pixels connected to the heart and $5 \%$ bad results especially due to a digestive artificial uptake. Using the inverse KarhnuenLoeve transform facilitated threshold and edge detection. Because the assumptions for the signal are few, this method can be easily applied others cardiac imaging methods such as PET, MRI or contrast ventriculography.
After method application, left ventricular ejection fraction, regional myocardial motion and thickening can be assessed. The next step is to compare this method with that described by Germano et al. using an asymmetrical Gaussien fit of the myocardial count distribution profile [6] and that proposed by Goris et al. using 3-D data and thresholding around three two dimensional vectors [7-8].

\section{Conclusion}

We propose an automatic myocardial contour detection procedure, which combines thresholding tools and morphological operations. This PC- based method can be used to improve the reproducibility of cardiac function quantification.

\section{Acknowledgements}

The authors thank the technical staff of the Department of Nuclear Cardiology of Jean Minjoz University Hospital and of the Saint Vincent Clinic for their expert assistance.

\section{References}

[1] DePuey EG, Rozanki AR. Using gated technetium-99m sestamibi SPECT to characterize fixed myocardial defects as infarct or artifact. J Nucl Med 1995; 36:52-4.

[2] Bateman TM, Berman DS, Heller GV, Brown KA, Cerqueira MD, Verani MS, Udelson JE. American Society of Nuclear Cardiology position statement on electrocardiographic gating of myocardial perfusion SPECT scintigrams. J Nucl Cardiol 1999; 6:470-2.

[3] Blagosklonov O, Sabbah R, Verdenet J, Cardot JC. Application of Karhunen-Loeve transform in nuclear cardiology : spatio-temporal smoothing and quantitative image analysis. Computer in Cardiology 2000; 27:299-4

[4] Davies E. Machine vision: Theory, Algorithms and Practicalities. Academic Press 1990:149-12.

[5] Pratt WK. Digital Image Processing. John Wiley \& Sons, Inc., 1991.

[6] Germano G, Kiat H, Kavanagh PB et al. Automatic quantification of ejection fraction from gated myocardial perfusion SPECT. J Nucl Med 1995; 36:2138-10.

[7] Goris ML, Thompson C, Malone LJ, Franken PR. Modelling the integration of myocardial regional perfusion and function. Nucl Med Commun 1994; 15:9-12.

[8] Everaert H, Franken PR, Flamen P, Goris M, Momen A, Bossuyt A. Left ventricular ejection fraction from gated SPET myocardial perfusion studies: a method based on the radial distribution of count rate density across the myocardial wall 1996; 23:1628-6.

Address for correspondence.

Name. COMAS Laurent

Address. Explorations cardiaques radio-isotopiques

Hôpital Jean MINJOZ 25030 BESANCON CEDEX, FRANCE

E-mail address.1comas@chu-besancon.fr 